\title{
Suzaku and XMM-Newton observations of the newly discovered early-stage cluster merger of 1E2216.0-0401 and 1E2215.7-0404
}

\author{
H. Akamatsu ${ }^{1}$, L. Gu${ }^{1}$, T. W. Shimwell ${ }^{2}$, F. Mernier ${ }^{1,2}$, J. Mao ${ }^{1,2}$, I. Urdampilleta ${ }^{1,2}$, \\ J. de Plaa $^{1}$, H. J. A. Röttgering ${ }^{2}$, and J. S. Kaastra ${ }^{1,2}$
}

\author{
${ }^{1}$ SRON Netherlands Institute for Space Research, Sorbonnelaan 2, 3584 CA Utrecht, The Netherlands \\ e-mail: h.akamatsu@sron.nl \\ 2 Leiden Observatory, Leiden University, PO Box 9513, 2300 RA Leiden, The Netherlands
}

Received 8 July 2016 / Accepted 3 August 2016

\begin{abstract}
We present the results of Suzaku and XMM-Newton X-ray observations of the cluster pair 1E2216.0-0401 and 1E2215.7-0404. We discover an X-ray bridge between the clusters. Suzaku and XMM-Newton observations revealed that each cluster hosts gas with moderate temperature of $k T_{0401}=4.8 \pm 0.1 \mathrm{keV}$ and $k T_{0404}=5.8 \pm 0.2 \mathrm{keV}$, respectively. On the other hand, the bridge region shows a remarkably high temperature $(k T=6.6 \pm 0.5 \mathrm{keV})$. Furthermore, at the position of the bridge, we detected an enhancement in the wavelet-decomposed soft-band $(0.5-4.0 \mathrm{keV})$ XMM-Newton image at 3 sigma significance, this is most likely due to a compression of the intracluster medium (ICM) as a consequence of the merging activity. This X-ray intensity and temperature enhancement are not consistent with those expected from a late phase, but are in agreement with the predictions by numerical simulations of an early phase merger. From the temperature jump at the location of the bridge, the Mach number is estimated to be $\mathcal{M}=1.4 \pm 0.1$, which corresponds to a shock propagation velocity of about $1570 \mathrm{~km} \mathrm{~s}^{-1}$. From the shock properties, we estimate that core-passage will occur in 0.3-0.6 Gyr and that the age of the shock structure is 50-100 Myr. Based on the measured properties of the ICM at the bridge and estimation of timescales, we find indications for non-equilibrium ionization. We also discover possible diffuse radio emission located between the merging clusters. Combining the radio, X-ray, and optical image data, we speculate that the detected radio sources are most likely related to the merger event. Thus, 1E2216.0-0401 and 1E2215.7-0404 is a new example of an early phase cluster merger with remarkable characteristics.
\end{abstract}

Key words. shock waves - galaxies: clusters: intracluster medium - X-rays: galaxies: clusters - radio continuum: galaxies galaxies: clusters: individual: 1E2216.0-0401 - galaxies: clusters: individual: 1E2215.7-0404

\section{Introduction}

According to the framework of hierarchical structure formation, galaxy clusters are the largest gravitationally bound objects in the Universe. Galaxy clusters are expected to grow through merging processes, that is, from collisions with smaller subhaloes such as sub-clusters and groups of galaxies. Such cluster merging processes release up to $\sim 10^{64} \mathrm{erg}$ of kinetic energy into several channels such as heating that is due to mergerinduced shocks, turbulence, and the amplification of the intergalactic magnetic field (Markevitch \& Vikhlinin 2007). These non-thermal phenomena might (re-)acceralate (pre-accelerated) particles up to relativistic energies, producing synchrotron radio emission (for a review, see e.g. Feretti et al. 2012). Combined $\mathrm{X}$-ray and radio observations therefore may help us in understanding the cluster merging process and related phenomena. Knowledge of the cluster energy partition of different components is crucial information not only to understand its role in cluster evolution (e.g. Voit 2005), but also to construct a cosmological probe (e.g. Allen et al. 2011).

Previously a handful of merging clusters were investigated in the X-ray band Markevitch et al. (1999, 2002), Finoguenov et al. (2010), Russell et al. (2010, 2012), Macario et al. (2011), Ogrean \& Brüggen (2013), Bourdin et al. (2013), Akamatsu \& Kawahara (2013) and also see Markevitch (2010). However, most of these observed mergers are in a late phase (after core-crossing) because the early phase (before core-crossing) is relatively shortlived (e.g. Ricker \& Sarazin 2001). Until now, there are a few representative examples of early-phase cluster mergers: A399 and A401 (Fujita et al. 1996, 2008), A1750 (Belsole et al. 2004; Bulbul et al. 2016), Cygnus A (Markevitch et al. 1999; Sarazin et al. 2013), and CIZA J1358.9-4750 (Kato et al. 2015). We do not include A222 and 223 because of their large line-of-sight distance difference $\sim 15-18 \mathrm{Mpc}$ (Werner et al. 2008; Dietrich et al. 2012). Although Kato et al. (2015) reported the signature of a merger shock in X-ray data, the other three early-phase cluster mergers do not show any clear shock structure. Furthermore, no concrete evidence of the connection between radio emission and such shocks has been reported so far. Consequently, the physics of early-phase cluster mergers remains poorly understood.

We here report a new example of an early-phase cluster merger based on the results of Suzaku and XMM-Newton observations of 1E2216.0-0401 (RA $=22 \mathrm{~h} 18 \mathrm{~m} 40.5 \mathrm{~s}$, Dec = $-03 d 46 m 48 s: z=0.09$; Cruddace et al. 2002) and 1E2215.7$0404(\mathrm{RA}=22 \mathrm{~h} 18 \mathrm{~m} 17.1 \mathrm{~s}$, Dec $=-03 \mathrm{~d} 50 \mathrm{m03s}: z=0.09$; Gioia $\&$ Luppino 1994). We refer to these clusters as 0401 and 0404 , respectively. The two clusters are separated by a projected distance of $640 \mathrm{kpc}(\sim 7.2 \mathrm{arcmin})$. We assume the cosmological parameters $H_{0}=70 \mathrm{~km} \mathrm{~s}^{-1} \mathrm{Mpc}^{-1}, \Omega_{\mathrm{M}}=0.27$ and $\Omega_{\Lambda}=0.73$. At a redshift of $z=0.09,1^{\prime}$ corresponds to $91.8 \mathrm{kpc}$. As our fiducial reference for the solar photospheric abundances denoted by $Z_{\odot}$, 


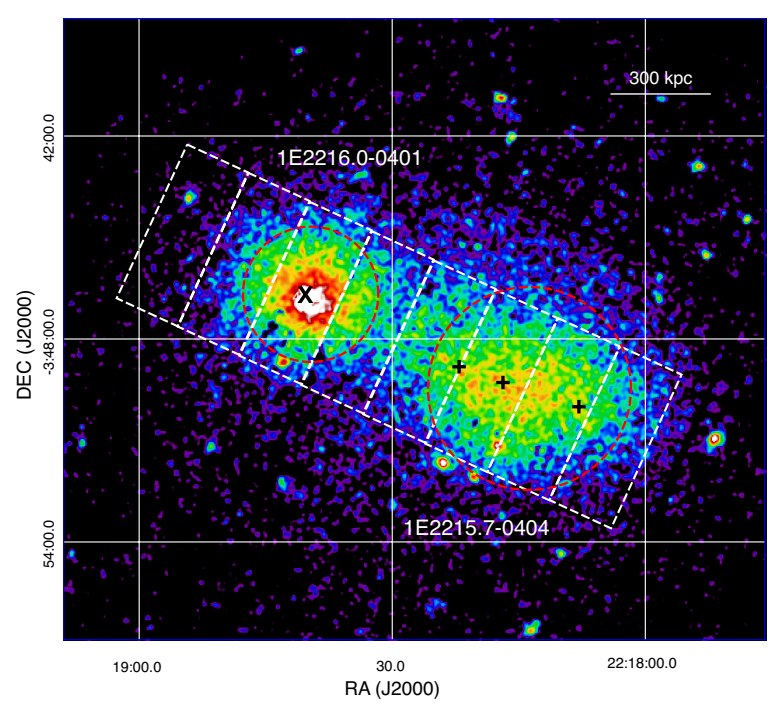

Fig. 1. Background-subtracted XMM-Newton image of 1E2216.00401 and 1E2215.7-0404 in the $0.8-8.0 \mathrm{keV}$ band. The magenta circles and white boxes $\left(2^{\prime} \times 5^{\prime}\right)$ show the regions that we discuss in this Letter. The $\times$ and + indicate the dominant galaxy(ies) of each cluster.

Table 1. Observational log.

\begin{tabular}{lllll}
\hline \hline & ID & $\begin{array}{l}\text { RA, Dec } \\
(\mathrm{J} 2000)\end{array}$ & $\begin{array}{l}\text { Observation } \\
\text { Starting date }\end{array}$ & $\begin{array}{l}\text { Exp. } \\
(\mathrm{ks})\end{array}$ \\
\hline Suzaku & 807085010 & $334.65,-3.76$ & $2012-05-16$ & 24.9 \\
& 807084010 & $334.58,-3.85$ & $2012-11-29$ & 11.2 \\
$X M^{a}$ & 677180101 & $334.57,-3.83$ & $2011-06-13$ & $10.5,13.5,6.7$ \\
\hline
\end{tabular}

Notes. ${ }^{(a)}$ Exposure times are for EPIC MOS1, 2 and pn.

we adopt those reported by Lodders et al. (2009). A Galactic absorption column of $N_{\mathrm{H}}=7.3 \times 10^{20} \mathrm{~cm}^{-2}$ (Willingale et al. 2013) was included in all fits. Unless otherwise stated, all our spectral analyses were made by using the Cash statistics (Cash 1979) and the errors correspond to $68 \%$ confidence for each parameter.

\section{Observations and data reduction}

Suzaku (Mitsuda et al. 2007) performed two observations of the two clusters (Fig. 1). The X-ray imaging sensor (XIS: Koyama et al. 2007) on board Suzaku consists of three front-side illuminated (FI) CCD chips (XIS0 and XIS3) and one back-side illuminated (BI) chip (XIS1). All observations were performed with either the normal $5 \times 5$ or $3 \times 3$ clocking mode. Data reduction was performed with HEAsoft version 6.15 and CALDB version 20140624. We started with the standard data screening provided by the Suzaku team and applied an event screening with geomagnetic cosmic-ray cut-off rigidity (COR2) $>6 \mathrm{GV}$ to suppress the detector background. An additional screening was applied for the XIS1 detector to minimize the detector background. We followed the processes described in the Suzaku XIS official document $^{1}$. The positions of the calibration sources and the failing area in XIS0 were excluded ${ }^{2}$. The resulting clean exposure times are 25 and $11 \mathrm{ks}$, respectively. The observational information is shown in Table 1.

\footnotetext{
1 http://www . astro.isas. jaxa.jp/suzaku/analysis/xis/ xis1_ci_6_nxb/

2 http://www.astro.isas.jaxa.jp/suzaku/doc/suzakumemo/ suzakumemo-2010-01.pdf
}

Table 2. Suzaku best-fit parameters for each cluster and for the bridge region.

\begin{tabular}{ccccc}
\hline \hline & $\begin{array}{c}k T \\
(\mathrm{keV})\end{array}$ & $\begin{array}{c}Z \\
\left(Z_{\odot}\right)\end{array}$ & $\begin{array}{c}\text { Norm } \\
\left(10^{70} / \mathrm{m}^{3} / \square^{\prime}\right)\end{array}$ & C-stat/d.o.f. \\
\hline 0401 & $4.75 \pm 0.10$ & $0.33 \pm 0.04$ & $31.3 \pm 0.5$ & $1130 / 1085$ \\
0404 & $5.68 \pm 0.18$ & $0.33 \pm 0.04$ & $18.1 \pm 0.8$ & $1230 / 1229$ \\
Bridge & $6.63 \pm 0.48$ & $0.3($ fix $)$ & $14.5 \pm 1.3$ & $342 / 311$ \\
\hline
\end{tabular}

XMM-Newton was used to observe 1E2216.0-0401 on 2011 June 13 for a short exposure of $15.9 \mathrm{ks}$. The SAS v13.5 and the built-in extended source analysis software (ESAS) were used to process and calibrate the data obtained with the XMM-Newton European Photon Imaging Camera (EPIC). Following a standard procedure, the MOS raw data were created by emchain, and the light curves were extracted and screened for time-variable background components by the mos-filter task. The final net clean exposures are $10.5 \mathrm{ks}, 13.5 \mathrm{ks}$, and $6.7 \mathrm{ks}$ for the MOS1, MOS2, and pn data, respectively. The observational information is shown in Table 1.

\section{Spectral analysis and result}

\subsection{Spectral analysis approach}

For the spectral analysis of Suzaku data, we used the SPEX (Kaastra et al. 1996) software version 3.01.00. Each spectrum was binned based on the optimal binning method (Kaastra \& Bleeker 2016). The best-fit parameters were obtained by minimizing the C-stat. In all the spectral analysis results presented here, the detector background (non X-ray background: NXB) was estimated from the night Earth data base using xisnxbgen (Tawa et al. 2008) and subtracted from the observed spectra. Other background components such as Galactic emission and unresolved X-ray sources (cosmic X-ray background, $\mathrm{CXB}$ ) were modelled during the fitting procedure. For the spectral fitting, we used the energy ranges of $0.8-8.0 \mathrm{keV}$ for both detectors. To estimate exactly the responses of the X-ray telescope and XIS, we employed the Monte Carlo simulator xissimarfgen (Ishisaki et al. 2007). As an input image, we employed a flat-field emission model $\left(r=20^{\prime}\right)$. For the Galactic background components, we used two thermal (the cie model in SPEX) components: one is unabsorbed and the other is absorbed. In both components, we fixed the abundance and the redshift to unity and zero, respectively. Furthermore, we fixed the temperature of each component to $0.08 \mathrm{keV}$ and $0.3 \mathrm{keV}$, respectively. For the fitting, we kept the normalization of thermal components free. For the CXB component, we refer to Kushino et al. (2002). In total, we used the spectral model $c i e+a b s *\left(c i e+\right.$ Powerlaw + cie $\left._{\mathrm{ICM}}\right)$. Because of the energy range we used for the fitting, our results are insensitive to the Galactic background components.

The XMM-Newton source spectra, NXB, and response files were prepared by the mos-spectra task. The CXB and two Galactic foreground components were calculated in the same way for the Suzaku analysis. To complement the limited angular resolution of the Suzaku XRT (HPD 2': Serlemitsos et al. 2007), we used XMM-Newton images to identify and evaluate the fluxes of point sources, which were detected by the cheese task above a flux threshold of $5 \times 10^{-14} \mathrm{ergs} \mathrm{cm}^{-2} \mathrm{~s}^{-1}$. These point sources were excluded in all the subsequent spectral analysis. 


\subsection{Results}

For the first step, to understand the global properties of each cluster, we extracted spectra from circular regions centred on 0401 and 0404 with $r=2^{\prime}$ and $r=3^{\prime}$, respectively. The bestfit values are listed in Table 2 . Both clusters show similar values for the temperature $\left(k T_{0401}=4.8 \pm 0.1 \mathrm{keV}, k T_{0404}=\right.$ $5.7 \pm 0.2 \mathrm{keV})$ and the abundance $\left(Z_{0401}=0.33 \pm 0.04 Z_{\odot}\right.$, $\left.Z_{0404}=0.33 \pm 0.04 Z_{\odot}\right)$. The redshifts derived for the clusters are $z_{0401}=0.093 \pm 0.001$ and $z_{0404}=0.091 \pm 0.001$. The velocity difference derived from the redshifts $\Delta v \sim 600 \mathrm{~km} \mathrm{~s}^{-1}$ is smaller than the sound speed (galaxy velocity dispersion) of each cluster $\left(c_{\mathrm{s}} \sim 1100 \mathrm{~km} \mathrm{~s}^{-1}\right)$. This means that the two clusters are at a similar line-of-sight distance.

To investigate the temperature structure in the direction of the merging axis (0401 to 0404), we extracted spectra in 8 boxes $\left(2^{\prime} \times 5^{\prime}: 182 \mathrm{kpc} \times 460 \mathrm{kpc}\right)$, as shown in Fig. 1 . We followed the same procedure as described above but fixed the abundance to $0.3 Z_{\odot}$ (e.g. Fujita et al. 2008). Considering the difference between the two solar abundance tables, the Fe abundance in this latter is 1.5 times higher than that of Fujita et al. (2008). To investigate the influence of uncertainties on the background components in the Suzaku data, we considered $30 \%$ and $3 \%$ fluctuations of the intensity of the CXB and the NXB components (Tawa et al. 2008). Because we did not use the low-energy band, the effect of the Galactic background is limited. Therefore, we did not consider this systematic error.

The resulting temperature profile is shown in Fig. 2, where red and blue crosses represent the best-fit value of Suzaku and $X M M-N e w t o n$, respectively. In general, the results of Suzaku and $X M M-N e w t o n$ are consistent with each other within the statistical uncertainties (68\% significance). The basic feature of the profile is insensitive to the systematic error of the background components (grey shallow region). Between both clusters $(r=$ $\left.3.0^{\prime}-5.0^{\prime}\right)$, the ICM shows a clear enhancement from $k T_{r=1^{\prime}-3^{\prime}}=$ $4.7 \mathrm{keV}$ in the cluster centres to $k T_{\text {Bridge,Suzaku }}=6.6 \mathrm{keV}$ in the region between the clusters. We also found an enhancement of the surface brightness in the $0.5-4.0 \mathrm{keV} \mathrm{XMM-Newton} \mathrm{image}$ (Figs. 2 and 3).

To detect enhanced ICM structure in the connecting region, we applied a wavelet decomposition based on the à Trous transform (e.g. $\mathrm{Gu}$ et al. 2009) on the vignetting-corrected $X M M$-Newton image. By masking out coefficients within $3 \sigma$ fluctuations of the background region and reconstructing the features with scales above $\sim 100 \mathrm{kpc}$, we obtained a significant coefficient map as shown in the right panel of Fig. 3. The observed features, such as the shock-heated and compressed region between the two clusters, agree well with predictions by numerical simulations (Takizawa 1999; Akahori \& Yoshikawa 2010).

The structure located between the clusters that is centred on the high-temperature region suggests the presence of a pressure discontinuity, that is, the presence of a shock front.

\section{Discussion}

A clear shock structure in an early-phase cluster merger has so far rarely been observed. To understand the nature of early-phase cluster mergers, additional examples are important.

The observational features revealed by Suzaku and $X M M-N e w t o n$ cannot be explained by the late phase of a merger. Furthermore, based on available optical information, we found some peculiar galaxies to be present in 0401 and 0404 . The positions of these galaxies are roughly consistent with the X-ray peak of each cluster (cross and plus in Fig. 1). This indicates

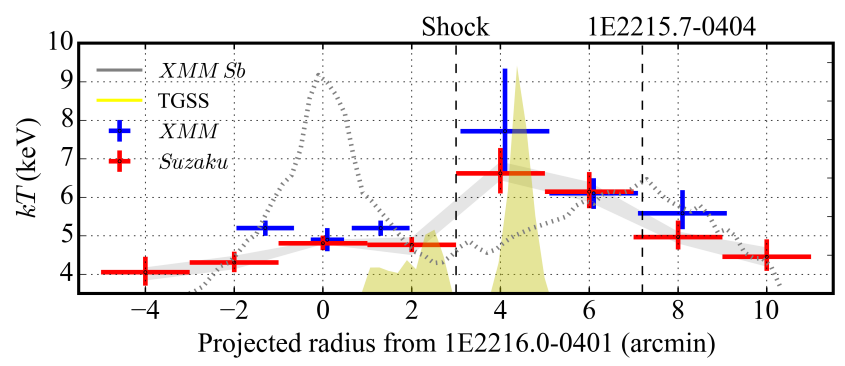

Fig. 2. Radial temperature profile of 1E2216.0-0401. Red and blue crosses show Suzaku and XMM-Newton results, respectively. The dashed line indicates the XMM-Newton $0.5-7.0 \mathrm{keV}$ surface brightness profile. The grey range represents the uncertainties of the Suzaku temperature estimation that is due to the combined 3\% variation of the NXB level and the $30 \%$ fluctuation in the CXB. The yellow region shows GMRT $154 \mathrm{MHz}$ radio emission. The bins of XMM-Newton are slightly offset $\left(0.1^{\prime}\right)$ for clarity.

that the ICM of each cluster has not been perturbed for a long time by the merger. Most likely the clusters yet have to enter core-crossing (early merger phase) but are close enough to interact and form a shock front.

The Suzaku and XMM-Newton temperature profiles show a clear jump at the bridge region, indicating the presence of a shock front. Here we evaluate the properties of this shock structure based on the Suzaku measurements alone because they have a lower particle background and longer observation time. The Mach number $\mathcal{M}$ can be estimated by applying the Rankine- Hugoniot jump condition (Landau \& Lifshitz 1959), $\frac{T_{2}}{T_{1}}=\frac{5 \mathcal{M}^{4}+14 \mathcal{M}^{2}-3}{16 \mathcal{M}^{2}}$ assuming the ratio of specific heats as $\gamma=5 / 3$, and 1 and 2 denote pre-shock and post-shock, respectively. Substituting the pre- and post-shock temperature $T_{1}=$ $4.7 \pm 0.2 \mathrm{keV}$ and $T_{2}=6.6 \pm 0.5 \mathrm{keV}$ into the above equation gives a Mach number $\mathcal{M}_{\text {Suzaku }}=1.4 \pm 0.1$. Following Eq. (2) in Markevitch et al. (1999), the shock compression $C$ can be estimated from the measured temperature ratio. Substituting the pre- and post-shock temperature ratio in the equation gives a shock compression $C \sim 1.56$. The heating due to adiabatic compression is expected to be about $T_{2}=T_{1} \cdot C^{\gamma-1} \sim 6.3 \mathrm{keV}$, in good agreement with the post-shock temperature. This means that the dominant part of the heating is due to adiabatic compression. The estimated low Mach number and heating mechanism are consistent with the properties of a shock structure that cosmological simulations predict to occur in an early-phase cluster merger (e.g. Miniati et al. 2000; Ricker \& Sarazin 2001; Ryu et al. 2003).

Combining the sound speed at the pre-shock region $\left(c_{\mathrm{s}} \sim\right.$ $1150 \mathrm{~km} \mathrm{~s}^{-1}$ ) with the Mach number, the shock propagation velocity is estimated to be about $1570 \mathrm{~km} \mathrm{~s}^{-1}$. With the assumption that the clusters move with the same velocity, we estimate that the core passing will occur in 0.3-0.6 Gyr. Under the same assumption, the age of the shock is also estimated to be 50-100 Myr.

Owing to the low-density environment at the cluster periphery, it is commonly believed that non-equilibrium ionization and the electron-ion temperature structure remain just after the shock because it has not had enough time to equilibrate (Takizawa \& Mineshige 1998; Rudd \& Nagai 2009; Akahori \& Yoshikawa 2010). The equilibration timescales of nonequilibrium ionization $t_{\mathrm{CIE}}$ and electron-ion two-temperature 

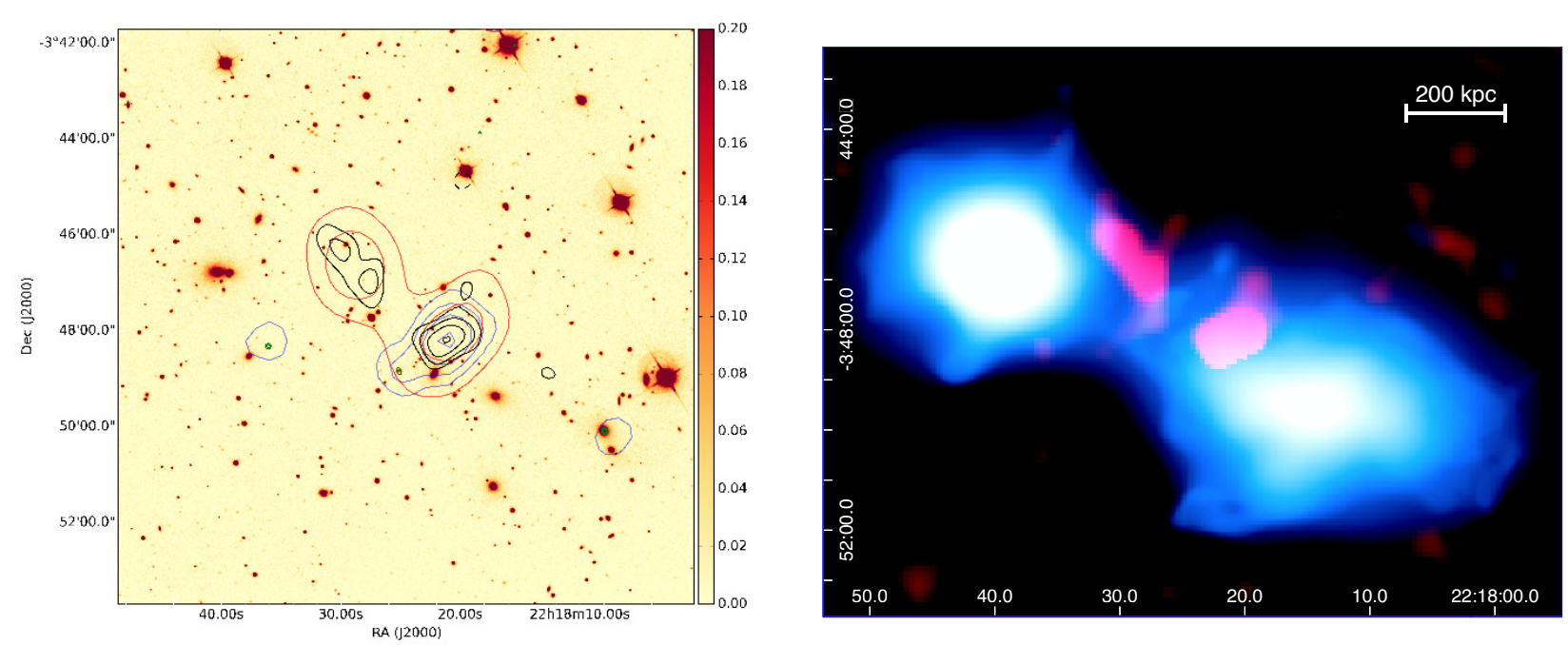

Fig. 3. Left: radio emission from VLSSr, TGSS, FIRST, and NVSS in red, black, green, and blue respectively. The contours are at $(1,2,4, \ldots) \times$ $4 \times \sigma$ levels where $\sigma$ is $90 \mathrm{mJy} /$ beam, $6.0 \mathrm{mJy} /$ beam, $0.24 \mathrm{mJy} /$ beam and $0.6 \mathrm{mJy} /$ beam for the VLSSr, TGSS, FIRST, and NVSS, respectively. The radio emission is overlaid on an SDSS $r$-band image of the region. Right: wavelet-decomposed 0.5-4.0 keV XMM-Newton image in blue. We show sources with $>3 \sigma$ significance and scales above $\sim 100 \mathrm{kpc}$. The filamentary structure between two halos is visible in the image. Red shows the $154 \mathrm{MHz}$ radio intensity from the TGSS alternative data release (Intema et al. 2016). Furthermore, there is a structure in the X-ray image at the bridge, which might be the shock-compressed ICM region.

structure $t_{\text {ie }}$ (Spitzer 1956; Masai 1984; Takizawa 1998) can be described as $t_{\mathrm{CIE}} \sim 3 \times 10^{9}\left(\frac{n_{\mathrm{e}}}{10^{-4} \mathrm{~cm}^{-3}}\right)^{-1} \mathrm{yr}$, $t_{\text {ie }} \sim 2 \times 10^{9}\left(\frac{n_{\mathrm{e}}}{10^{-4} \mathrm{~cm}^{-3}}\right)^{-1}\left(\frac{T}{10^{8} \mathrm{~K}}\right)^{\frac{3}{2}} \mathrm{yr}$, respectively. Here, we estimate the electron density of the bridge region from the cie normalization (norm $=n_{\mathrm{e}} n_{\mathrm{X}} V$ ). Assuming a $2 \mathrm{Mpc}$ line-of-sight depth and the normalization value of the unit area (Table 2), the electron density at the shock-heated region is estimated to be $n_{\mathrm{e}}=6.0 \times 10^{-4}(l / 2 \mathrm{Mpc})^{-0.5} \mathrm{~cm}^{3}$, where $l$ is the line-of-sight depth. We note that $l$ is quite unconstrained, hence $n_{\mathrm{e}}$ is rather uncertain. Combined with the assumption of $l=2 \mathrm{Mpc}$ and the measured shock temperature, the equilibration timescales are estimated to be $t_{\mathrm{CIE}} \sim 0.5 \mathrm{Gyr}$ and $t_{\mathrm{ie}} \sim 0.2 \mathrm{Gyr}$. These timescales are longer than the estimated age of the shock structure estimated from the shock propagation velocity, indicating that a part of the shock-heated region has not yet reached equilibrium.

To investigate the possibility of a non-equilibrium ionization state, we evaluated the $r t$ parameter in the cie model, which represents the ratio of ionization balance temperature to electron temperature. The resulting value $\left(r t=0.45_{-0.10}^{+0.38}\right)$ suggests that the ICM at the shock region has not yet reached ionization equilibrium, which will lead to an underestimation of the postshock temperature. Furthermore, we note that Suzaku's large PSF might lead to a similar effect through the effects of smearing. Therefore, the estimated Mach number is a lower limit We stress that it is a challenge to firmly conclude about nonequilibrium ionization states with the currently available X-ray data. Deep X-ray observations are strongly desired. Future highresolution X-ray spectrometers such as the Athena X-IFU instrument (Ravera et al. 2014) will enable us not only to investigate these non-equilibrium states, but will also make detailed spectroscopic diagnostics possible (e.g. Kaastra et al. 2009).

Diffuse radio emission is observed to be associated with the ICM of approximately 100 galaxy clusters and is generally thought to be formed as a consequence of shocks and turbulence in massive clusters (see e.g. Feretti et al. 2012 and Brunetti \& Jones 2014, for recent reviews). To search for such diffuse radio emission from the ICM of this merging cluster system and to characterize it as a function of frequency and resolution we have used the following wide-area radio surveys: the $74 \mathrm{MHz}$ VLA Low-Frequency Sky Survey (VLSSr; Lane et al. 2012), the $150 \mathrm{MHz}$ TIFR GMRT Sky Survey alternative data release (TGSS; Intema et al. 2016), the 1.4 GHz NRAO/VLA Sky Survey (NVSS; Condon et al. 1998), and the 1.4 GHz Faint Radio Images of the Sky at Twenty-Centimeters (FIRST; Becker et al. 1995).

We find that two main regions of diffuse radio emission are located in the vicinity of 0401 and 0404 that are approximately coincident with the X-ray observed bridge between the clusters. These are object $\mathrm{A}$ at 22:18:22 -03:48:08 and object B at 22:18:29 -03:46:42. From the radio survey images we estimate that for object $\mathrm{A}$ the $150 \mathrm{MHz}$ integrated brightness is $570 \pm 60 \mathrm{mJy}$ and the $74 \mathrm{MHz}$ to $1.4 \mathrm{GHz}$ spectral index is $-1.2 \pm 0.1$, while for object $\mathrm{B}$ the integrated $150 \mathrm{MHz}$ brightness is $350 \pm 50 \mathrm{mJy}$ and the $74 \mathrm{MHz}$ to $150 \mathrm{MHz}$ spectral index is $-2.0 \pm 0.3$ (object $\mathrm{B}$ is not detected in the $1.4 \mathrm{GHz}$ images). Steep spectral indices together with the lack of an obvious optical counterpart or compact radio core (see Fig. 3 left) are properties that are expected for radio emission that is associated with or influenced by shocks and turbulence in the ICM. Furthermore, such a classification is supported by the clear merging nature of the system and the close proximity of the radio emission and the $\mathrm{X}$-ray detected shock. However, before any clear classification of this radio emission is made it is important that more sensitive and higher resolution radio observations are conducted to thoroughly assess the morphological, spectral, and polarization properties of the emission.

We showed that 1E2216.0-0401 and 1E2215.7-0404 are a new example of an early-phase cluster merger with a clear signature of a merger-induced shock front and possibly diffuse radio emission, which may also be related to the merging activity. Additional investigation with the high spatial and spectral resolution X-ray observatories (XMM-Newton, Chandra and also Athena), sensitive radio observations (GMRT, JVLA, and LOFAR), weak-lensing mass maps, and sophisticated numerical 
simulations will provide us with deeper insight of the cluster merger dynamics and the origin of the diffuse radio emission.

Acknowledgements. The authors wish to thank the referee for constructive comments that improved the manuscript. The authors thank the Suzaku and XMMNewton team members for their support on the projects. H.A acknowledges the support of NWO via a Veni grant. SRON is supported financially by NWO, the Netherlands Organization for Scientific Research.

\section{References}

Akahori, T., \& Yoshikawa, K. 2010, PASJ, 62, 335

Akamatsu, H., \& Kawahara, H. 2013, PASJ, 65, 16

Allen, S. W., Evrard, A. E., \& Mantz, A. B. 2011, ARA\&A, 49, 409

Becker, R. H., White, R. L., \& Helfand, D. J. 1995, ApJ, 450, 559

Belsole, E., Pratt, G. W., Sauvageot, J.-L., \& Bourdin, H. 2004, A\&A, 415, 821

Bourdin, H., Mazzotta, P., Markevitch, M., Giacintucci, S., \& Brunetti, G. 2013, ApJ, 764, 82

Brunetti, G., \& Jones, T. W. 2014, Int. J. Mod. Phy. D, 23, 30007

Bulbul, E., Randall, S. W., Bayliss, M., et al. 2016, ApJ, 818, 131

Cash, W. 1979, ApJ, 228, 939

Condon, J. J., Cotton, W. D., Greisen, E. W., et al. 1998, AJ, 115, 1693

Cruddace, R., Voges, W., Böhringer, H., et al. 2002, ApJS, 140, 239

Dietrich, J. P., Werner, N., Clowe, D., et al. 2012, Nature, 487, 202

Feretti, L., Giovannini, G., Govoni, F., \& Murgia, M. 2012, A\&ARv, 20, 54

Finoguenov, A., Sarazin, C. L., Nakazawa, K., Wik, D. R., \& Clarke, T. E. 2010 ApJ, 715, 1143

Fujita, Y., Koyama, K., Tsuru, T., \& Matsumoto, H. 1996, PASJ, 48, 191

Fujita, Y., Tawa, N., Hayashida, K., et al. 2008, PASJ, 60, 343

Gioia, I. M., \& Luppino, G. A. 1994, ApJS, 94, 583

Gu, L., Xu, H., Gu, J., et al. 2009, ApJ, 700, 1161

Intema, H. T., Jagannathan, P., Mooley, K. P., \& Frail, D. A. 2016, A\&A, submitted [arXiv: 1603.04368]

Ishisaki, Y., Maeda, Y., Fujimoto, R., et al. 2007, PASJ, 59, 113

Kaastra, J. S., \& Bleeker, J. A. M. 2016, A\&A, 587, A151

Kaastra, J. S., Mewe, R., \& Nieuwenhuijzen, H. 1996, in UV and X-ray Spectroscopy of Astrophysical and Laboratory Plasmas, eds. K. Yamashita, \& T. Watanabe, 411
Kaastra, J. S., Bykov, A. M., \& Werner, N. 2009, A\&A, 503, 373

Kato, Y., Nakazawa, K., Gu, L., et al. 2015, PASJ, 67, 71

Koyama, K., Tsunemi, H., Dotani, T., et al. 2007, PASJ, 59, 23

Kushino, A., Ishisaki, Y., Morita, U., et al. 2002, PASJ, 54, 327

Landau, L. D., \& Lifshitz, E. M. 1959, Fluid mechanics (Oxford: Pergamon Press)

Lane, W. M., Cotton, W. D., Helmboldt, J. F., \& Kassim, N. E. 2012, Radio Science, 47, RS0K04

Lodders, K., Palme, H., \& Gail, H.-P. 2009, in Landolt Börnstein, Vol. VI/4B, Chap. 4.4, 560

Macario, G., Markevitch, M., Giacintucci, S., et al. 2011, ApJ, 728, 82

Markevitch, M. 2010, ArXiv e-prints [arXiv: 1010.3660]

Markevitch, M., \& Vikhlinin, A. 2007, Phys. Rep., 443, 1

Markevitch, M., Sarazin, C. L., \& Vikhlinin, A. 1999, ApJ, 521, 526

Markevitch, M., Gonzalez, A. H., David, L., et al. 2002, ApJ, 567, L27

Masai, K. 1984, Ap\&SS, 98, 367

Miniati, F., Ryu, D., Kang, H., et al. 2000, ApJ, 542, 608

Mitsuda, K., Bautz, M., Inoue, H., et al. 2007, PASJ, 59, 1

Ogrean, G. A., \& Brüggen, M. 2013, MNRAS, 433, 1701

Ravera, L., Barret, D., den Herder, J. W., et al. 2014, in Space Telescopes and Instrumentation 2014: Ultraviolet to Gamma Ray, Proc. SPIE, 9144, 91442L

Ricker, P. M., \& Sarazin, C. L. 2001, ApJ, 561, 621

Rudd, D. H., \& Nagai, D. 2009, ApJ, 701, L16

Russell, H. R., Sanders, J. S., Fabian, A. C., et al. 2010, MNRAS, 406, 1721

Russell, H. R., McNamara, B. R., Sanders, J. S., et al. 2012, MNRAS, 423, 236

Ryu, D., Kang, H., Hallman, E., \& Jones, T. W. 2003, ApJ, 593, 599

Sarazin, C. L., Finoguenov, A., \& Wik, D. R. 2013, Astron. Nachr., 334, 346

Serlemitsos, P. J., Soong, Y., Chan, K.-W., et al. 2007, PASJ, 59, 9

Spitzer, L. 1956, Physics of Fully Ionized Gases (New York: Interscience Publishers)

Takizawa, M. 1998, ApJ, 509, 579

Takizawa, M. 1999, ApJ, 520, 514

Takizawa, M., \& Mineshige, S. 1998, ApJ, 499, 82

Tawa, N., Hayashida, K., Nagai, M., et al. 2008, PASJ, 60, 11

Voit, G. M. 2005, Rev. Mod. Phys., 77, 207

Werner, N., Finoguenov, A., Kaastra, J. S., et al. 2008, A\&A, 482, L29

Willingale, R., Starling, R. L. C., Beardmore, A. P., Tanvir, N. R., \& O'Brien, P. T. 2013, MNRAS, 431,394 